\title{
HOMOMORPHISM COMPLEXES AND K-CORES
}

\author{
GREG MALEN
}

\begin{abstract}
AвSTRACT. We prove that the topological connectivity of a graph homomorphism complex $\operatorname{Hom}\left(G, K_{m}\right)$ is at least $m-D(G)-2$, where $D(G)=\max _{H \subseteq G} \delta(H)$. This is a strong generalization of a theorem of Čukić and Kozlov, in which $D(G)$ is replaced by the maximum degree $\Delta(G)$. It also generalizes the graph theoretic bound for chromatic number, $\chi(G) \leq D(G)+1$, as $\chi(G)=\min \left\{m: \operatorname{Hom}\left(G, K_{m}\right) \neq \varnothing\right\}$. Furthermore, we use this result to examine homological phase transitions in the random polyhedral complexes $\operatorname{Hom}\left(G(n, p), K_{m}\right)$ when $p=c / n$ for a fixed constant $c>0$.
\end{abstract}

\section{INTRODUCTION}

The study of graph homomorphism complexes, $\operatorname{Hom}(G, H)$ for fixed graphs $G$ and $H$, grew out of a rising interest in finding topological obstructions to graph colorings, as in Lovász's proof of the Kneser Conjecture [11]. Specifically, Lovász used the topological connectivity of the Neighborhood Complex, $\mathcal{N}(G)$, to provide a lower bound on the chromatic number of a Kneser graph. It turns out that $\mathcal{N}(G)$ is a specialization of a homomorphism complex, as it is homotopy equivalent to $\operatorname{Hom}\left(K_{2}, G\right)$ (see Proposition 4.2 in [2]). The structure of the underlying graphs has many quantifiable effects on the topology of these complexes. The 0 -cells of $\operatorname{Hom}(G, H)$ are precisely the graph homomorphisms from $G \rightarrow H$, and so the most straighforward such result is that the chromatic number of $G, \chi(G)$, is the minimum $m$ for which $\operatorname{Hom}\left(G, K_{m}\right)$ is non-empty. For $\Delta(G)$ the maximum degree of $G$, Babson and Kozlov conjectured [2], and Čukić and Kozlov later proved [6] that if $\Delta(G)=d$, then $\operatorname{Hom}\left(G, K_{m}\right)$ is at least $(m-d-2)$-connected. Note that when $m=d+1$ this recovers the well known bound $\chi(G) \leq \Delta(G)+1$.

In graph theory, there is an improvement of this bound using the minimum degree of induced subgraphs, namely that $\chi(G) \leq \max _{H \subseteq G} \delta(H)+1$, for $\delta(H)$ the minimum degree of $H$. The value $D(G):=\max _{H \subseteq G} \delta(H)$ is known as the degeneracy of $G$, a graph property which is commonly utilized in computer science and the study of large networks. Using $D(G)$ in place of $\Delta(G)$, in Section 3 we prove a generalization of this graph theoretic result which will also specialize to the Čukić-Kozlov Theorem. In Section 4 we focus on the case that $H=K_{3}$ and show, with only a few notable exceptions, that $\operatorname{Hom}\left(G, K_{3}\right)$ is disconnected if it is non-empty.

Furthermore, in the setting of random graphs the evolution of $D(G)$ for a sparse ErdôsRényi graph $G \sim G(n, p)$ has been well studied. For $p=c / n$ for a constant $c>0$, Pittel, Spencer, and Wormwald exhibited sharp thresholds for the appearance and size of subgraphs with minimum degree $k$ [13]. In Section 5 we combine our results with their work to exhibit both phase transitions and lower bounds for the topological connectivity of the random polyhedral complexes $\operatorname{Hom}\left(G(n, c / n), K_{m}\right)$ for constant $c>0$. When $m=3$,

Date: October 10, 2018. 
we are able to use the results from Section 4 to evaluate the limiting probability for the connectivity of $\operatorname{Hom}\left(G(n, c / n), K_{3}\right)$ for all $c>0$.

\section{Background AND Definitions}

In the following, all graphs are undirected simple graphs, and $K_{m}$ is the complete graph on $m$ vertices. For a graph $G$ and a vertex $v \in V(G)$, when it is clear from context we may write simply that $v \in G$. In this section we define a graph homomorphism complex, or homcomplex, and various properties which will be used to acquire bounds on the topological connectivity of these complexes.

Definition 2.1. For fixed graphs $G$ and $H$, a graph homomorphism from $G \rightarrow H$ is an edge preserving map on the vertices, $f: V(G) \rightarrow V(H)$ such that $\{f(v), f(u)\} \in E(H)$ if $\{v, u\} \in E(G)$.

Definition 2.2. For fixed graphs $G$ and $H$, cells in $\operatorname{Hom}(G, H)$ are functions

$$
\eta: V(G) \rightarrow 2^{V(H)} \backslash \varnothing
$$

with the restriction that if $\{x, y\} \in E(G)$, then $\eta(x) \times \eta(y) \subseteq E(H)$. The dimension of $\eta$ is defined to be $\operatorname{dim}(\eta)=\sum_{v \in V(G)}(|\eta(v)|-1)$, with ordering $\eta \subseteq \tau$ if $\eta(v) \subseteq \tau(v)$ for all $v \in V(G)$.

The set of 0-cells of $\operatorname{Hom}(G, H)$ is then precisely the set of graph homomorphisms from $G \rightarrow H$, with higher dimensional cells formed over them as multihomomorphisms. Furthermore, every cell in a hom-complex is a product of simplices, and hom-complexes are entirely determined by their 1-skeletons. If $\eta$ is a product of simplices and its 1-skeleton is contained in $\operatorname{Hom}(G, H)$, then $\eta \in \operatorname{Hom}(G, H)$. The 1-skeleton of $\operatorname{Hom}(G, H)$ can be thought of in the following manner. Two graph homomorphisms $\eta, \tau: G \rightarrow H$ are adjacent in $\operatorname{Hom}(G, H)$ if and only if their images differ on exactly one vertex $v \in G$. So $\eta(v) \neq \tau(v)$, $\eta(u)=\tau(u)$ for all $u \in G \backslash\{v\}$, and the 1-cell joining $\eta$ and $\tau$ is the multihomomorphism $\sigma$ for which $\sigma(v)=\eta(v) \cup \tau(v)$ and $\sigma(u)=\eta(u)=\tau(u)$ for all $u \in G \backslash\{v\}$.

Since the property of being a homomorphism is independent on disjoint connected components of a graph, hom-complexes obey a product rule for disjoint unions. For any graphs $G, G^{\prime}$ and $H$,

$$
\operatorname{Hom}\left(G \amalg G^{\prime}, H\right)=\operatorname{Hom}(G, H) \times \operatorname{Hom}\left(G^{\prime}, H\right)
$$

Thus, when convenient we can always restrict our attention to connected graphs. Here we also introduce the notion of a graph folding.

Definition 2.3. Denote the neighborhood of a vertex $v \in G$ by $N(v)=\{w \in G: w \sim v\}$. Let $v, u \in G$ be distinct vertices such that $N(v) \subseteq N(u)$. Then a fold of $G$ is a homomorphism from $G \rightarrow G \backslash\{v\}$ which sends $v \mapsto u$ and is otherwise the identity.

Lemma 2.4. (Babson, Kozlov; Proposition 5.1 in [2]). If $G$ and $H$ are graphs and $v, u \in G$ are distinct vertices such that $N(v) \subseteq N(u)$, then the folding $G \rightarrow G \backslash\{v\}$ which sends $v \mapsto u$ induces a homotopy equivalence $\operatorname{Hom}(G \backslash\{v\}, H) \rightarrow \operatorname{Hom}(G, H)$.

If $T$ is a tree, for example, then $T$ folds to a single edge, $\operatorname{so} \operatorname{Hom}\left(T, K_{m}\right) \simeq \operatorname{Hom}\left(K_{2}, K_{m}\right)$. And by Proposition 4.5 in [2], $\operatorname{Hom}\left(K_{n}, K_{m}\right)$ is homotopy equivalent to a wedge of $(m-n)$ spheres, and in particular $\operatorname{Hom}\left(K_{2}, K_{m}\right) \simeq S^{m-2}$. $\operatorname{So} \operatorname{Hom}\left(T, K_{m}\right) \simeq S^{m-2}$ for any tree $T$. For a thorough introduction to hom-complexes, see [2]. 
Notation 2.5. For a graph $G$ define the maximum degree to be $\Delta(G):=\max _{v \in V(G)}\{\operatorname{deg}(v)\}$, and the minimum degree to be $\delta(G):=\min _{v \in V(G)}\{\operatorname{deg}(v)\}$.

Definition 2.6. The $k$-core of a graph $G$ is the subgraph $c_{k}(G) \subseteq G$ obtained by the process of deleting vertices with degree less then $k$, along with all incident edges, one at a time until there are no vertices with degree less than $k$.

Regardless of the order in which vertices are deleted, this process always terminates in the unique induced subgraph $c_{k}(G) \subseteq G$ which is maximal over all subgraphs of $G$ which have minimum degree at least $k$. The $k$-core of a graph may be the empty graph, and the existence of a non-empty $k$-core is a monotone question, as $c_{l}(G) \subseteq c_{j}(G)$ whenever $j \leq l$. Here we are interested in the most highly connected non-trivial subgraph.

Definition 2.7. The degeneracy of $G$ is $D(G):=\max _{H \subseteq G} \delta(H)$, for $H$ an induced subgraph.

Then $D(G)$ is the maximum $k$ such that $c_{k}(G)$ is non-empty. Given these definitions, we are now able to state the main theorem:

Theorem 2.8. For any graph $G, \operatorname{Hom}\left(G, K_{m}\right)$ is at least $(m-D(G)-2)$-connected.

And $D(G) \leq \Delta(G)$, hence this will imply the C̆ukić-Kozlov Theorem. Our interest is primarily in applying this result to the case that $G$ is a sparse Erdős-Rényi random graph, where $D(G)$ has been studied extensively and is much smaller than $\Delta(G)$. It should also be noted that Engström gave a similar strengthening of the Čukić-Kozlov Theorem in [8], replacing $\Delta(G)$ with a graph property which is independent of $D(G)$ and which may provide a better tool for studying the dense regime.

\section{Proof of Theorem 2.8}

To prove the theorem, we first introduce a result of Csorba which finds subcomplexes that are homotopy equivalent to $\operatorname{Hom}\left(G, K_{m}\right)$ by removing independent sets of $G$.

Notation 3.1. Let $G$ and $H$ be graphs. Define

$$
\operatorname{Ind}(G):=\{S \subseteq V(G): S \text { is an independent set }\}
$$

And for $I \in \operatorname{Ind}(G)$ define

$$
\operatorname{Hom}_{I}(G, H):=\left\{\eta \in \operatorname{Hom}(G \backslash I, H): \text { there is } \bar{\eta} \in \operatorname{Hom}(G, H) \text { with } \bar{\eta}_{G \backslash I}=\eta\right\}
$$

So $\operatorname{Hom}_{I}(G, H)$ is the subcomplex of $\operatorname{Hom}(G \backslash I, H)$ comprised of all multihomomorphisms from $G \backslash I \rightarrow H$ which extend to multihomomorphisms from $G \rightarrow H$.

Theorem 3.2. (Csorba; Theorem 2.36 in [5]) For any graphs $G$ and $H$, and any $I \in \operatorname{Ind}(G)$, $\operatorname{Hom}(G, H)$ is homotopy equivalent to $\operatorname{Hom}_{I}(G, H)$.

The proof of this is an application of both the Nerve Lemma and the Quillen Fiber Lemma, and a more thorough examination of this property is given by Schultz in [14]. Note that when $H=K_{m}$ and $I=\{v\}$ for any vertex $v \in G$, a multihomomorphism $\eta: G \backslash\{v\} \rightarrow K_{m}$ 
has an extension $\bar{\eta}: G \rightarrow K_{m}$ as long as there is some vertex in $K_{m}$ which is not in $\eta(w)$ for any $w \in N(v)$. Thus

$$
\operatorname{Hom}_{\{v\}}\left(G, K_{m}\right)=\left\{\eta \in \operatorname{Hom}\left(G \backslash\{v\}, K_{m}\right):\left|\bigcup_{w \in N(v)} \eta(w)\right| \leq m-1\right\}
$$

Lemma 3.3. Let $G$ be a graph with a vertex $v$ such that $\operatorname{deg}(v) \leq k$. Then the $(m-k-1)$ skeleton of $\operatorname{Hom}\left(G \backslash\{v\}, K_{m}\right)$ is contained in $\operatorname{Hom}_{\{v\}}\left(G, K_{m}\right)$.

Proof of Lemma 3.3. Let $\eta \in \operatorname{Hom}\left(G \backslash\{v\}, K_{m}\right) \backslash \operatorname{Hom}_{\{v\}}\left(G, K_{m}\right)$. Then

$$
m=\left|\bigcup_{w \in N(v)} \eta(w)\right| \leq \sum_{w \in G \backslash\{v\}}|\eta(w)|
$$

And

$$
\operatorname{dim}(\eta)=\sum_{w \in G \backslash\{v\}}(|\eta(w)|-1)=\left(\sum_{w \in G \backslash\{v\}}|\eta(w)|\right)-|N(v)| \geq m-k
$$

Hence if $\eta \in \operatorname{Hom}\left(G \backslash\{v\}, K_{m}\right)$ with $\operatorname{dim}(\eta) \leq m-k-1$, then $\eta \in \operatorname{Hom}_{\{v\}}\left(G, K_{m}\right)$. $\square$

So if $\operatorname{Hom}\left(G \backslash\{v\}, K_{n}\right)$ is $(m-k-2)$-connected, then $\operatorname{Hom}_{\{v\}}\left(G, K_{n}\right)$ is $(m-k-2)$ connected. Combining this with Csorba's theorem, we have the following corollary and the proof of Theorem 2.8:

Corollary 3.4. Let $G$ be a graph with a vertex v such that $\operatorname{deg}(v) \leq k$. If $\operatorname{Hom}\left(G \backslash\{v\}, K_{n}\right)$ is $(m-k-2)$-connected, then $\operatorname{Hom}\left(G, K_{n}\right)$ is $(m-k-2)$-connected.

Proof of Theorem 2.8. Let $G$ be a graph with $D(G)=k$. Then $c_{k+1}(G)$ is the empty graph, and there is a sequence $G=G_{0}, G_{i}=G_{i-1} \backslash\left\{v_{i}\right\}$, with $\operatorname{deg}_{G_{i-1}}\left(v_{i}\right) \leq k$, terminating in $G_{|V(G)|}=\varnothing$. So $G_{|V(G)|-1}$ is a single vertex, and $\operatorname{Hom}\left(G_{|V(G)|-1}, K_{n}\right)=\Delta^{m}$, the $m$-simplex, which is contractible, and thus $(m-k-2)$-connected. Hence, by induction, $\operatorname{Hom}\left(G, K_{m}\right)$ is also $(m-k-2)$-connected.

\section{4. $\operatorname{Hом}\left(G, K_{3}\right)$}

When $G$ is a graph such that $\chi(G) \leq 3, \operatorname{Hom}\left(G, K_{3}\right)$ has a particularly nice structure. If $G$ does not have an isolated vertex, then $\operatorname{Hom}\left(G, K_{3}\right)$ is a cubical complex, and Babson and Kozlov showed that it admits a metric with nonpositive curvature [2]. Notice that for a connected graph $G$ with $\chi(G) \leq 3$, the bound obtained by Theorem 2.8 when $m=3$ provides no new information. When $D(G)=1, G$ is a tree and folds to a single edge, so $\operatorname{Hom}\left(G, K_{3}\right) \simeq \operatorname{Hom}\left(K_{2}, K_{3}\right) \simeq S^{1}$. When $D(G)=2$, the bound merely confirms that $\operatorname{Hom}\left(G, K_{3}\right)$ is non-empty, and for $D(G)>2$ it gives no information at all. Here we show that $\operatorname{Hom}\left(G, K_{3}\right)$ is, in fact, disconnected for a large class of graphs $G$ with $\chi(G) \leq 3$.

Theorem 4.1. If $G$ is a graph with $\chi(G)=3$, then $\operatorname{Hom}\left(G, K_{3}\right)$ is disconnected.

This result has been formulated previously in the language of statistical physics, where Glauber dynamics examines precisely the 1-skeleton of $\operatorname{Hom}\left(G(n, p), K_{m}\right)$. See, for example, the work of Cereceda, van den Heuvel and Johnson [3]. We provide a proof here in the context of work on hom-complexes of cycles done by C̆ukić and Kozlov, and offer a 
more general approach for lifting disconnected components via subgraphs in the following lemma.

Lemma 4.2. Let $G \subseteq G^{\prime}$ and $H$ be graphs such that $\operatorname{Hom}(G, H)$ is non-empty and disconnected. Let $\eta_{1}, \eta_{2} \in \operatorname{Hom}(G, H)$ be 0-cells, i.e. graph homomorphisms from $G \rightarrow H$, such that they are in distinct connected components of $\operatorname{Hom}(G, H)$. If there are extensions $\overline{\eta_{1}}, \overline{\eta_{2}} \in \operatorname{Hom}\left(G^{\prime}, H\right)$ with $\left.\bar{\eta}_{i}\right|_{G}=\eta_{i}$ for $i \in\{1,2\}$, then $\operatorname{Hom}\left(G^{\prime}, H\right)$ is also disconnected.

Proof of Lemma 4.2. Let $\eta_{1}, \eta_{2}$ be graph homomorphisms from $G \rightarrow H$ such that they are in distinct connected components of $\operatorname{Hom}(G, H)$, with extensions $\overline{\eta_{1}}, \overline{\eta_{2}} \in \operatorname{Hom}\left(G^{\prime}, H\right)$. We may assume that $\operatorname{dim}\left(\bar{\eta}_{i}\right)=0$ for each $i$, since otherwise any 0 -cells they contain would also be extensions of $\eta_{i}$. Suppose that $\overline{\eta_{1}}, \overline{\eta_{2}}$ are in the same connected component of $\operatorname{Hom}\left(G^{\prime}, H\right)$. Then there is a path $\overline{\eta_{1}}=\overline{\tau_{0}} \sim \overline{\tau_{1}} \sim \overline{\tau_{2}} \sim \ldots \sim \overline{\tau_{l}}=\overline{\eta_{2}}$ in $\operatorname{Hom}\left(G^{\prime}, H\right)$, with $\operatorname{dim}\left(\overline{\tau_{j}}\right)=0$ for all $j$. Let $\tau_{j}=\left.\overline{\tau_{j}}\right|_{G}$, for $0 \leq j \leq l$. Then as functions on $V\left(G^{\prime}\right)$, for each $j$ there is one vertex $v_{j} \in G^{\prime}$ for which $\overline{\tau_{j}}\left(v_{j}\right) \neq \overline{\tau_{j+1}}\left(v_{j}\right)$, and they agree on all other vertices. If $v_{j} \in G^{\prime} \backslash G$, then for the restrictions $\tau_{j}=\tau_{j+1}$. If $v_{j} \in G$, then $\tau_{j}\left(v_{j}\right) \neq \tau_{j+1}\left(v_{j}\right)$, but they agree on all other vertices, so $\tau_{j} \sim \tau_{j+1}$ in $\operatorname{Hom}(G, H)$. Hence the path in $\operatorname{Hom}\left(G^{\prime}, H\right)$ projects onto a possibly shorter path from $\eta_{1}$ to $\eta_{2}$ in $\operatorname{Hom}(G, H)$, which is a contradiction.

Therefore the extensions $\overline{\eta_{1}}$ and $\overline{\eta_{2}}$ must be in different connected components of $\operatorname{Hom}\left(G^{\prime}, H\right)$, which is thus disconnected.

The subgraphs we examine to obtain disconnected components of $\operatorname{Hom}\left(G, K_{3}\right)$ will be cycles. In [7], Čukić and Kozlov fully characterized the homotopy type of $\operatorname{Hom}\left(C_{n}, C_{m}\right)$ for all $n, m \in \mathbb{N}$. In particular, they showed that all 0 -cells in a given connected component have the same number of what they call return points. For these complexes, let $V\left(C_{n}\right)=$ $\left\{v_{1}, \ldots, v_{n}\right\}$ such that $v_{i} \sim v_{(i+1) \bmod n}$ for all $1 \leq i \leq n$, and let $V\left(C_{m}\right)=\{1, \ldots, m\}$ such that $j \sim(j+1) \bmod m$ for all $1 \leq j \leq m$. For the purpose of defining the return number of a 0 -cell $\eta$, we momentarily drop the set bracket notation and write $\eta\left(v_{i}\right)=j$.

Definition 4.3. A return point of a 0 -cell $\eta \in \operatorname{Hom}\left(C_{n}, C_{m}\right)$ is a vertex $v_{i} \in C_{n}$ such that $\eta\left(v_{i+1}\right)-\eta\left(v_{i}\right) \equiv-1 \bmod m$. Then $r(\eta)$, the return number of $\eta$, is the number of $v_{i} \in C_{n}$ which are return points of $\eta$.

Note that since $\eta$ is a homomorphism, the quantity $\eta\left(v_{i+1}\right)-\eta\left(v_{i}\right)$ is always $\pm 1 \bmod m$. It simply measures in which direction $C_{n}$ is wrapping around $C_{m}$ on the edge $\left\{v_{i}, v_{i+1}\right\}$.

Lemma 4.4. ( $\breve{C u k i c ́, K o z l o v, ~ L e m m a ~} 5.3$ in [7]) If two 0-cells of Hom $\left(C_{n}, C_{m}\right)$ are in the same connected component, then they have the same return number.

For a 0 -cell $\eta \in \operatorname{Hom}\left(G, K_{m}\right)$, one may always obtain another 0 -cell by swapping the inverse images of two vertices in $K_{m}$. When the target graph is a complete graph, we refer to the inverse image of a vertex in $K_{m}$ as a color class of $\eta$. When $m=3$, we have $K_{3}=C_{3}$ and we can track the effect that interchanging two color classes has on the return number.

Notation 4.5. For a 0 -cell $\eta \in \operatorname{Hom}\left(G, K_{m}\right)$, let $\eta_{\{l, j\}}$ denote the $(l, j)$-interchanging 0 -cell obtained by defining $\eta_{\{l, j\}}^{-1}(l)=\eta^{-1}(j), \eta_{\{l, j\}}^{-1}(j)=\eta^{-1}(l)$, and $\eta_{\{l, j\}}^{-1}(i)=\eta^{-1}(i)$ for all $i \notin\{l, j\}$.

Lemma 4.6. Fix a pair $\{l, j\} \subset\{1,2,3\}, l \neq j$. Then for any 0 -cell $\eta \in \operatorname{Hom}\left(C_{n}, K_{3}\right)$, every $v_{i} \in C_{n}$ is a return point for exactly one of $\eta$ and $\eta_{\{l, j\}}$. Hence $r\left(\eta_{\{l, j\}}\right)=n-r(\eta)$. 
Proof of Lemma 4.6. Consider the edge $\left\{v_{i}, v_{i+1}\right\}$. SInce the target graph is $K_{3}$, we have that $\left\{\eta\left(v_{i}\right), \eta\left(v_{i+1}\right)\right\} \cap\{l, j\} \neq \varnothing$. If $\left\{\eta\left(v_{i}\right), \eta\left(v_{i+1}\right)\right\}=\{l, j\}$, then

$$
\eta_{\{l, j\}}\left(v_{i+1}\right)-\eta_{\{l, j\}}\left(v_{i}\right)=-\left(\eta\left(v_{i+1}\right)-\eta\left(v_{i}\right)\right)
$$

Thus $v_{i}$ is a return point of $\eta$ if and only if it is not a return point of $\eta_{\{l, j\}}$. Alternatively, if $\left|\left\{\eta\left(v_{i}\right), \eta_{(} v_{i+1}\right\} \cap\{l, j\}\right|=1$, then one of $v_{i}$ and $v_{i+1}$ has a stationary image under an $(l, j)$ interchange while the other does not. By fixing the image of one vertex and changing the other, this flips the direction that $C_{n}$ is wrapping around $K_{3}$ on the edge $\left\{v_{i}, v_{i+1}\right\}$. So returns become non-returns and vice versa. Therefore each $v_{i} \in C_{n}$ is a return point of exactly one of $\eta$ and $\eta_{\{l, j\}}$ for any fixed $\{l, j\} \subset\{1,2,3\}$.

The proof of Theorem 4.1 will then proceed by finding an odd cycle in $G$ and using a color class interchange to produce 0 -cells in distinct connected components, which can be lifted from $\operatorname{Hom}\left(C_{2 k+1}, K_{3}\right)$ to $\operatorname{Hom}\left(G, K_{3}\right)$.

Proof of Theorem 4.1 Since $\chi(G)=3$, $\operatorname{Hom}\left(G, K_{3}\right)$ is non-empty and $G$ contains an odd cycle $H=C_{2 k+1}$ for some $k \in \mathbb{N}$. Note that we do not require $H$ to be an induced cycle. Let $\eta \in \operatorname{Hom}\left(G, K_{3}\right)$ be a 0 -cell, and let $\tau=\left.\eta\right|_{H}$ be the induced homomorphism on $H$. Fix a distinct pair $\{l, j\} \subset\{1,2,3\}$ and consider the $(l, j)$-interchange $\eta_{\{l, j\}}$. It is straightforward that $\left.\eta_{\{l, j\}}\right|_{H}=\tau_{\{l, j\}} \in \operatorname{Hom}\left(H, K_{3}\right)$. Thus by Lemma 4.6, $r\left(\tau_{\{l, j\}}\right)=2 k+$ $1-r(\tau) \neq r(\tau)$. Hence, by lemma 4.4 they are in different connected components of $\operatorname{Hom}\left(H, K_{3}\right)$, and by Lemma 4.2 their extensions $\eta$ and $\eta_{\{l, j\}}$ are in different connected components of $\operatorname{Hom}\left(G, K_{3}\right)$, which is thus disconnected. $\square$

Bipartite graphs are a bit more complicated. We have seen that for any graph $G$ which folds to a single edge, $\operatorname{Hom}\left(G, K_{3}\right) \simeq S^{1}$. When $G$ is bipartite and does not fold to an edge, it must contain an even cycle $C_{2 k}$ for $k \geq 3$. So by the same method one can start with $\eta \in \operatorname{Hom}\left(G, K_{3}\right)$ and take its restriction $\left.\eta\right|_{C_{2 k}}=\tau \in \operatorname{Hom}\left(C_{2 k}, K_{3}\right)$. But if $r(\tau)=k$, then interchanging two color classes no longer guarantees disjoint components. In particular, the existence of too many 4-cycles close to $C_{2 k}$ may force $r(\tau)=k$. For example, for $Q_{3}$ the 1-skeleton of the 3-cube, $\operatorname{Hom}\left(Q_{3}, K_{3}\right)$ is connected. Note that $D\left(Q_{3}\right)=3$, so the lower bound achieved by Theorem 2.8 does not provide any information. Restricting to the case that $G$ omits the subgraphs in Figure 4.1 is sufficient to ensure that this does not happen, and that $\operatorname{Hom}\left(G, K_{3}\right)$ is disconnected.
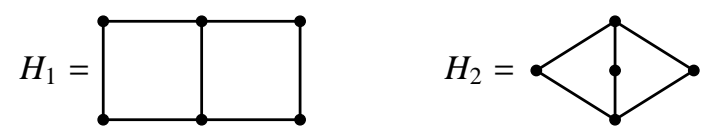

FIGURE 4.1. If $\operatorname{Hom}\left(G, K_{3}\right)$ is connected, then $G$ must contain $H_{1}$ or $H_{2}$.

Theorem 4.7. If $G$ is a finite, bipartite, connected graph which does not fold to an edge and does not contain $H_{1}$ or $H_{2}$ as a subgraph, then $\operatorname{Hom}\left(G, K_{3}\right)$ is disconnected.

To show this requires a great deal of case-by-case structural analysis when a minimal even cycle in $G$ has length 6,8 , or 10 . The method of the proof will suggest a stronger, albeit more technical result. 
Proof of Theorem 4.7. Let $G$ be a finite, bipartite, connected graph which does not fold to an edge and does not contain $H_{1}$ or $H_{2}$ as a subgraph. Since $G$ is finite, bipartite and does not fold to an edge, it must contain an even cycle of length greater than 4 . Let $H=C_{2 k}, k \geq 3$ be a fixed cycle in $G$ which has minimal length over all cycles in $G$ of length greater than 4. Label the vertices of $H$ as $v_{1}, \ldots, v_{2 k}$ such that $v_{i} \sim v_{(i+1) \bmod 2 k}$ for all i. $H$ cannot have internal chords, as any such edge would create a cycle $C_{l}$ for $4<l<2 k$, or in the case that $k=3$ an antipodal chord would create a copy of $H_{1}$. For $2 k \equiv i \bmod 3$, take $\tau \in \operatorname{Hom}\left(H, K_{3}\right)$ such that $\tau\left(v_{j}\right)=\{j \bmod 3\}$ for $j \leq 2 k-i$, with $\tau\left(v_{2 k}\right)=\{2\}$ if $i=1$, and $\tau\left(v_{2 k-1}\right)=\{1\}, \tau\left(v_{2 k}\right)=\{2\}$ if $i=2$. Figure 4.2 depicts $\tau$ for $k \in\{3,4,5\}$.

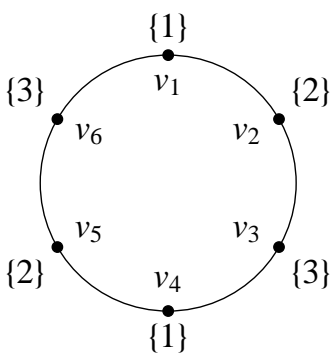

(a) $C_{6}, 2 k \equiv 0 \bmod 3$

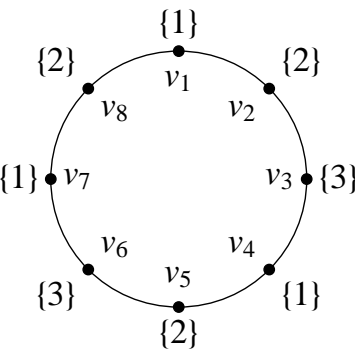

(b) $C_{8}, 2 k \equiv 2 \bmod 3$

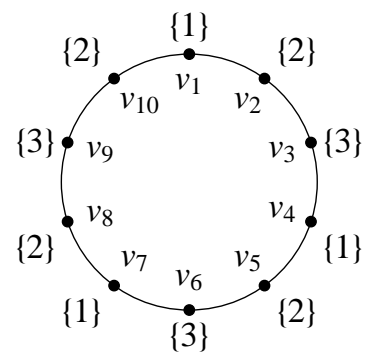

(c) $C_{10}, 2 k \equiv 1 \bmod 3$

FIGURE 4.2. Image of $\tau$ on $H=C_{2 k}$

As defined, only the last two vertices of $C_{2 k}$ can be return points of $\tau$. So $r(\tau) \leq 2$ in all cases, and hence is not equal to $k$ for any $k \geq 3$. Then for any $\{l, j\} \subset\{1,2,3\}$, $r\left(\tau_{\{l, j\}}\right) \neq r(\tau)$. Thus $\tau_{\{l, j\}}$ and $\tau$ are in distinct connected components of $\operatorname{Hom}\left(H, K_{3}\right)$. So if we can construct an extension $\eta$ to all of $G$, then $\eta_{\{l, j\}}$ will extend $\tau_{\{l, j\}}$ and hence $\eta$ and $\eta_{\{l, j\}}$ will be in distinct connected component of $\operatorname{Hom}\left(G, K_{3}\right)$.

Define $d(u, v)$ to be the minimal number of edges in a path connecting two vertices $u, v \in G$. Let $\sigma$ be a bipartition of $G$, viewed as a 0 -cell of $\operatorname{Hom}\left(G, K_{3}\right)$ with image contained in $\{1,2\}$. We will let $\eta=\sigma$ on $G \backslash H$ and then make adjustments as necessary when the definition conflicts with $\tau$. Define the following sets:

$$
\begin{aligned}
& B_{i}:=\{u \in G: \min \{d(u, v): v \in H\}=i\} \\
& A \quad:=\left\{u \in B_{1}: \exists v \in N(u) \cap H \text { with } \sigma(u)=\tau(v)\right\} \\
& A_{i, j}:=\{u \in A:|N(u) \cap H|=i,|N(u) \cap A|=j\}
\end{aligned}
$$

So $B_{i}$ is the set of points distance $i$ from $H$, and $A$ is the set of points in $G \backslash H$ on which we cannot define $\eta=\sigma$. To arrive at an appropriate definition for $\eta$ on the set $A$, we must first make some structural observations concerning the partitioning of $A$ into the subsets $A_{i, j}$.

Claim 4.8. For every $u \in B_{1},|N(u) \cap H| \leq 2$. And if $|N(u) \cap H|=2$, then $N(u) \cap H=$ $\left\{v_{i}, v_{(i+2) \bmod 2 k}\right\}$ for some $i \leq 2 k$.

Proof of Claim 4.8. Let $u \in B_{1}$. Then connecting $u$ to any two points in $H$ creates a cycle of length at most $k+2<2 k$, as in Figure 4.3 (a). Since $G$ is bipartite and $H$ is minimal with respect to having length at least 6 , this is valid only if it creates a 4-cycle, with 
$N(u) \cap H=\left\{v_{i}, v_{(i+2) \bmod 2 k}\right\}$ for some $i \leq 2 k$. Similarly, any further points of attachment would need to be in a 4-cycle with each of the other two vertices in $N(u) \cap H$. But this is only possible if $H=C_{6}$, in which case it would create several copies of $H_{1}$, shown in Figure 4.3 (b). ㅁ

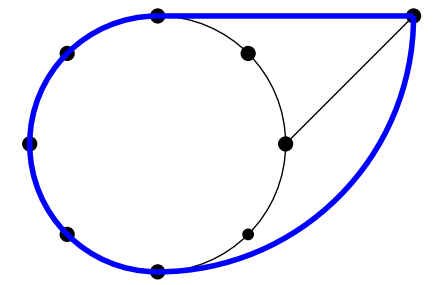

(a) $H=C_{8},|N(u) \cap H|>2 \Rightarrow C_{6}$

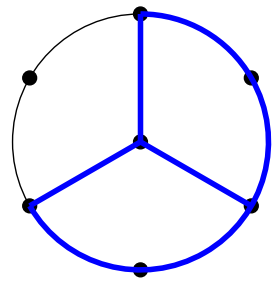

(b) $H=C_{6},|N(u) \cap H|>2 \Rightarrow H_{1}$

FIGURE 4.3. Obstructions to $|N(u) \cap H|>2$

This imposes that $i \leq 2$ for $A_{i, j} \subseteq A$, while the following two claims will show that $j$ is at most 1 , and that $j=1$ only if $i=1$.

Claim 4.9. Let $k \geq 4$, and let $u \in B_{1}$. Then $\left|N(u) \cap B_{1}\right| \leq 1$, and if $N(u) \cap B_{1} \neq \varnothing$ then $|N(u) \cap H|=1$.

Proof of Claim. Let $k \geq 4$, let $u \in B_{1}$, and let $w \in N(u) \cap B_{1}$, with $u \sim v_{i}$ and $w \sim v_{j}$. $G$ is bipartite, so $v_{i} \neq v_{j}$. If $v_{i} \nsim v_{j}$, as in Figure 4.4 (a), there is a cycle of length $l$ with $4<l \leq k+3<2 k$. So $v_{i} \sim v_{j}$. Suppose further that $|N(u) \cap H|=2$. Then $N(u) \cap H=\left\{v_{(j-1) \bmod 2 k}, v_{(j+1) \bmod 2 k}\right\}$ since both must be adjacent to $v_{j}$, as in Figure 4.4 (b). But then the induced graph on $\left\{u, w, v_{(j-1) \bmod 2 k}, v_{j}, v_{(j+1) \bmod 2 k}\right\}$ is a copy of $H_{2}$. So if $N(u) \cap B_{1} \neq \varnothing$ for $u \in B_{1}$, then $|N(u) \cap H|=1$. Now suppose $\left|N(u) \cap B_{1}\right|>1$. Then there is a vertex $x \in N(u) \cap B_{1}$ with $x \neq w$. As with $w$, the vertex in $N(x) \cap H$ must be adjacent to $v_{i}$. If $x \sim v_{j}$, as in Figure 4.4 (c), then $\left\{u, w, x, v_{i}, v_{j}\right\}$ is a copy of $H_{2}$, and if $x$ is connected to the other neighbor of $v_{i}$, as in Figure 4.4 (d), then it creates a copy of $H_{1}$. Thus $\left|N(u) \cap B_{1}\right| \leq 1$.

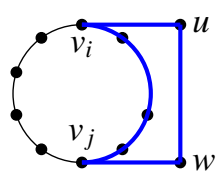

(a) $v_{i}+v_{j}$; $C_{k+3}$

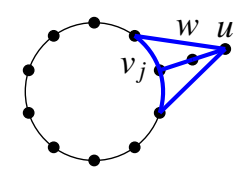

(b) $|N(u) \cap H|=2$; $\mathrm{H}_{2}$

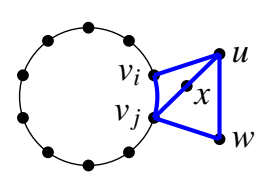

(c) $\left|N(u) \cap B_{1}\right|=2$; $\mathrm{H}_{2}$

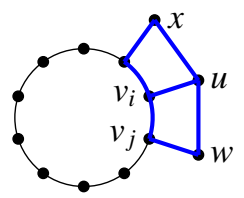

(d) $\left|N(u) \cap B_{1}\right|=2$; $H_{1}$

Figure 4.4. Invalid structures in $G$ if Claim 4.9 is false.

For $k=3$ a similar result holds when $B_{1}$ is replaced by the subset $A$ :

Claim 4.10. Let $k=3$, and let $u \in A$. Then $|N(u) \cap A| \leq 1$, and if $N(u) \cap A \neq \varnothing$ then $|N(u) \cap H|=1$. 
Proof of Claim. Let $u \in A$ and let $w \in N(u) \cap A$. Recall that for $2 k=6, \tau\left(v_{i}\right)=\{i \bmod 3\}$ for all $i$. Then without loss of generality assume that $\sigma(u)=\{1\}$ and $u \sim v_{1}$. So $\sigma(w)=\{2\}$, and since $w \in A$ it must be adjacent to at least one of $v_{2}$ and $v_{5}$. But $G$ is bipartite and $u \sim v_{5}$ creates a 5-cycle, as indicated in Figure 4.5 (a). Thus $w \sim v_{2}$, and the same argument holds to show that $u \sim v_{1}$ if one first assumes that $w \sim v_{2}$. Furthermore, if $w \sim v_{4}$ or $w \sim v_{6}$ there is a copy of $H_{1}$ or $H_{2}$, respectively, depicted in Figure 4.5 (b), (c). By symmetry $u \nsim v_{3}, u \nsim v_{5}$. Hence, if $u \in A$ with $N(u) \cap A \neq \varnothing$, then $|N(u) \cap H|=1$. Now suppose there is $x \in N(u) \cap A$ with $x \neq w$. Then $\sigma(x)=\{2\}$, and by the preceding argument we have that $x \sim v_{2}$. But, as shown in Figure 4.5 (d), $\left\{u, w, x, v_{1}, v_{2}\right\}$ is then a copy of $H_{2}$. Hence for $u \in A,|N(u) \cap A| \leq 1$.

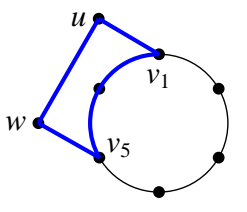

(a) $w \sim v_{5} ; C_{5}$

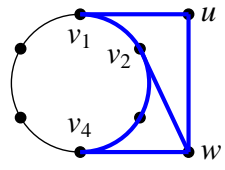

(b) $w \sim v_{4} ; H_{1}$

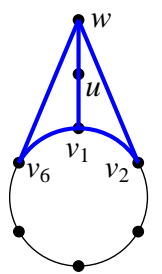

(c) $w \sim v_{6} ; H_{2}$

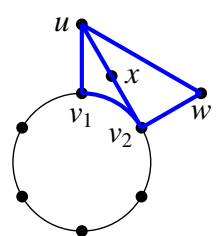

(d) $|N(u) \cap A|>2 ; H_{2}$

Figure 4.5. Invalid structures in $G$ if Claim 4.10 is false.

Then for all $k \geq 3$ we have that $A=A_{1,0} \cup A_{2,0} \cup A_{1,1}$, and vertices in $A_{1,1}$ which are adjacent to each other have adjacent attaching vertices in $H$. When possible, we will define $\eta(v)=\{3\}$ for vertices $v \in A$. However, this not possible on all vertices in $A_{1,1}$, nor for vertices in $A_{2,0}$ which have a neighbor in $H$ that is already assigned \{3\}. So define

$$
\bar{A}=\left\{u \in A_{2,0}:\{\tau(v): v \in N(u) \cap H\}=\{\sigma(u),\{3\}\}\right\} \cup\left\{u \in A_{1,1}: \sigma(u)=\{2\}\right\}
$$

Now define $\eta: G \rightarrow K_{3}$ by:

$$
\eta(u)=\left\{\begin{array}{ll}
\tau(u) & \text { if } u \in H \\
\{1\} & \text { if } u \in \bar{A} \cap A_{1,1} ; \\
\{2\} & \text { if } u \in \bar{A} \cap \cap \cap A_{2,0} \text { with } \sigma(u)=\{2\} \\
\{3\} & \text { if } u \in A \backslash \bar{A} ; \\
& u \in G \backslash(A \cup H) \text { with } N(u) \cap \bar{A} \neq \varnothing \\
\sigma(v) & \text { else }
\end{array}\right\}
$$

Notice that for $u \in \bar{A}, \eta(u)=\{1,2\} \backslash \sigma(u)=\sigma(w)$ for any $w \in N(u)$. Hence the last qualifier assigns $\{3\}$ to any neighbors of vertices in $\bar{A}$ which need to be switched, and $\eta=\sigma$ on all remaining vertices.

It is clear that this defines a homomorphism on $A \cup H$, and on any edges between vertices $u \in G \backslash(A \cup H)$ for which $N(u) \cap \bar{A}=\varnothing$. Thus it suffices to check that this is consistent on edges with a vertex $u \in G \backslash(A \cup H)$ for which $N(u) \cap \bar{A} \neq \varnothing$. Note that by definition, any such $u$ will have $\eta(u)=\{3\}$. So to ensure that $\eta(w) \neq\{3\}$ for any $w \in N(u)$, 
we must check that $N(u) \cap(A \backslash \bar{A})=\varnothing$, and for all $w \in N(u)$ that $N(w) \cap \bar{A}=\varnothing$. The cases $k=3$ and $k \geq 4$ must again be handled separately.

Claim 4.11. Let $k \geq 4$, and let $u \in G \backslash(A \cup H)$ with $x \in N(u) \cap \bar{A}$. Then $N(u) \cap(A \backslash \bar{A})=\varnothing$, and if $w \in N(u)$ then $N(w) \cap \bar{A}=\varnothing$.

Proof of Claim. Let $u$ and $x$ be as in the claim. Since $x \in A \subseteq B_{1}$, it must be that $u \in\left(B_{1} \cup B_{2}\right) \backslash A$. Suppose first that $u \in B_{1}$. Then $\left|N(u) \cap B_{1}\right| \leq 1$ by Claim 4.9, and hence $N(u) \cap A=\{x\}$. So $N(u) \cap(A \backslash \bar{A})=\varnothing$. Similarly, Claim 4.9 implies that $N(x) \cap B_{1}=\{u\}$ and that $|N(x) \cap H|=1$, so $x \in A_{1,0}$, which contradicts our assumption that $x \in \bar{A} \subseteq A_{2,0} \cup A_{1,1}$. So $u \notin B_{1}$.

Suppose instead that $u \in B_{2}$, and thus $N(u) \subseteq B_{1} \cup B_{2} \cup B_{3}$. For $w \in N(u) \cap B_{3}$ it is immediate that $w \notin A$ and that $(N(w) \cap \bar{A}) \subseteq\left(N(w) \cap B_{1}\right)=\varnothing$. Then assume that there is $w \in N(u) \cap B_{1}, w \neq x$. Then $\sigma(w)=\sigma(x)$ since $G$ is bipartite, and thus for any attaching points in $H, v_{i} \sim x, v_{j} \sim w$, it must be that $i$ and $j$ are either both odd or both even. Since $x \in A_{2,0} \cup A_{1,1}$ we may assume that either $i \neq j$ or $x \in A_{1,1}$. Recall that an adjacent pair in $A_{1,1}$ forms a square with its adjacent pair in $H$. So if $i=j$ and $x \in A_{1,1}$ with $\{z\}=N(x) \cap A_{1,1}$, then $z$ is adjacent to one of the two vertices in $N\left(v_{i}\right) \cap H$. As shown in Figure 4.6 (a), this creates a copy of $H_{1}$. So we may assume that $i \neq j$. But then the short path between $v_{i}$ and $v_{j}$ in $H$ along with $\{u, w, x\}$ creates a cycle of length $l, 6 \leq l \leq k+4$.

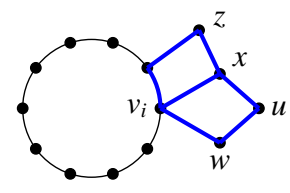

(a) $i=j, x \in A_{1,1} ; H_{1}$

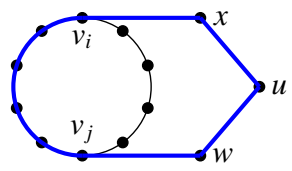

(b) $i \neq j$; $C_{9}$ for $k=5$

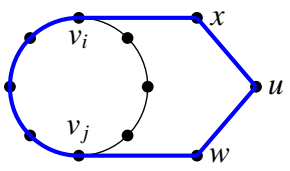

(c) $i \neq j ; C_{8}$ for $k=4$

FigURE 4.6. $w \in N(u) \cap B_{1}, w \neq x$.

If $k \geq 5$ this length is less than $2 k$, as in Figure 4.6 (b), which contradicts the minimality of $H$. If $k=4$, this creates a cycle of length 8 only if $j=(i+4) \bmod 8$, as in Figure 4.6 (c). Recall that as defined for $k=4$ (see Figure $4.2(\mathrm{~b})), \tau\left(v_{i}\right) \neq \tau\left(v_{(i+4) \bmod 8}\right)$ for all $i$. But $\sigma(x)=\sigma(w)$, and therefore $x$ and $w$ cannot both be in $A$. As this is true for any pair of vertices in $N(u) \cap B_{1}$, it must be that $|N(u) \cap A| \leq 1$, and hence by assumption $N(u) \cap A=\{x\}$. Furthermore, for any $w \in B_{1} \backslash A$, if $w \sim z$ with $z \in A$, then by Claim 4.9 it must be that $z \in A_{1,0}$. Thus $N(w) \cap \bar{A}=\varnothing$ for all $w \in N(u) \cap B_{1}$.

Now suppose that $w \in N(u) \cap B_{2}$. Note that $w \notin A \subseteq B_{1}$. Then let $x=x_{u}$ and let $x_{w} \in N(w) \cap B_{1}$. Note that $\sigma\left(x_{u}\right) \neq \sigma\left(x_{w}\right)$ since there is a path of length 3 from $x_{u}$ to $x_{w}$, so the connecting vertices for $x_{u}$ and $x_{w}$ in $H$ must be distinct to avoid creating an odd cycle. Then the path between their connecting vertices in $H$ along with $x_{u}, u, w$, and $x_{w}$ creates a cycle of length $l, 6 \leq l \leq k+5$. For $k>5$ such a cycle contradicts the minimality of $H$.

Suppose $k=4$. Then to avoid creating a cycle of length less than 8 , this construction requires that the path in $H$ between the connecting points of $x_{u}$ and $x_{w}$ is length 3. Suppose by way of contradiction that $x_{w} \in \bar{A}$. So both $x_{u}, x_{w} \in \bar{A} \subseteq A_{2,0} \cup A_{1,1}$. Without loss of 
generality, assume that $x_{u} \in A_{2,0}$ with $N\left(x_{u}\right) \cap H=\left\{v_{i}, v_{(i+2) \bmod 8}\right\}$ for some $1 \leq i \leq 8$. Then $v_{(i+5) \bmod 8}$ is the only vertex in $H$ which is distance 3 from both $v_{i}$ and $v_{(i+2) \bmod 8 \text {. }}$. So $x_{w}$ cannot also be in $A_{2,0}$, and thus $x_{w} \in A_{1,1}$ with $N\left(w_{x}\right) \cap H=\left\{v_{(i+5) \bmod 8}\right\}$. By definition, $\sigma(v)=\{2\}$ for all $v \in \bar{A} \cap A_{1,1}$, so we have that $\sigma\left(x_{w}\right)=\{2\}$, and thus also $\tau\left(v_{(i+5) \bmod 8}\right)=\{2\}$. For $\tau$ as defined, this forces $i \in\{3,5,8\}$. But now $\sigma\left(x_{u}\right)=\{1\}$, and so $x_{u} \in \bar{A} \cap A_{2,0}$ implies that $\left\{\tau\left(v_{i}\right), \tau\left(v_{(i+2)} \bmod 8\right)\right\}=\{\{1\},\{3\}\}$, which is only possible if $i \in\{1,4\}$. Figure 4.7 (a) and (b) shows the arrangements if $i=1$ and $i=4$ respectively, with $\tau\left(v_{6}\right), \tau\left(v_{1}\right) \neq\{2\}$. Hence it must be that both $x_{u}, x_{w} \in A_{1,1}$. However, $\sigma\left(x_{u}\right) \neq \sigma\left(x_{w}\right)$, and as noted above $\sigma(v)=\{2\}$ for all $v \in \bar{A} \cap A_{1,1}$. So at most one of $x_{u}$ and $x_{w}$ can be in $\bar{A}$, and by assumption $x_{u} \in \bar{A}$. Thus $x_{w} \notin \bar{A}$ and $N(w) \cap \bar{A}=\varnothing$ for all $w \in N(u) \cap B_{2}$.

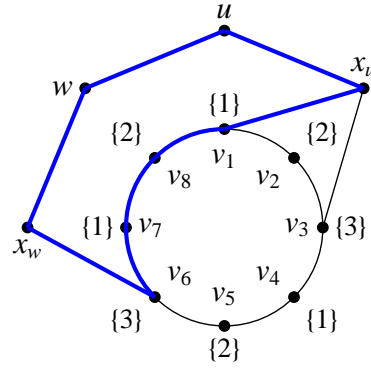

(a) $i=1, \tau\left(v_{6}\right) \neq\{2\}$

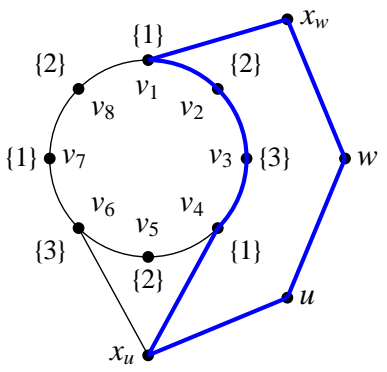

(b) $i=4, \tau\left(v_{1}\right) \neq\{2\}$

FiguRE 4.7. $x_{u} \in \bar{A} \cap A_{2,0}, x_{w} \in \bar{A} \cap A_{1,1}, \sigma\left(x_{u}\right)=\{1\}$ when $k=4$.

Now suppose $k=5$, and again assume that $x_{w} \in \bar{A}$. Here cycles created by adjoining $u, w, x_{u}$, and $x_{w}$ will be of length 10 only if the connecting vertices in $H=C_{10}$ are distance 5 apart. Hence neither $x_{u}$ nor $x_{w}$ can be in $A_{2,0}$, and both must be in $A_{1,1}$. Thus, as in the case above for $k=4, \sigma\left(x_{u}\right) \neq \sigma\left(x_{w}\right)$ implies that at most one of $x_{u}$ and $x_{w}$ can be in $\bar{A}$. Therefore $x_{w} \notin \bar{A}$ and $N(w) \cap \bar{A}=\varnothing$ for all $w \in N(u) \cap B_{2}$. $\square$

Claim 4.12. Let $k=3$, and let $u \in G \backslash(A \cup H)$ with $x \in N(u) \cap \bar{A}$. Then $N(u) \cap(A \backslash \bar{A})=\varnothing$, and if $w \in N(u)$ then $N(w) \cap \bar{A}=\varnothing$.

Proof of Claim. Let vertices $u$ and $x$ be as in the claim, and let $v_{i} \in N(x) \cap H$ be a vertex such that $\sigma(x)=\tau\left(v_{i}\right)$, which exists by the assumption that $x \in A$. Then since $G$ is bipartite, it must be that $\{v \in H: d(v, u)=2\} \subseteq\left\{v_{i}, v_{(i+2) \bmod 6}, v_{(i+4) \bmod 6}\right\}$. Recall that $\tau$ is defined such that for each $j \in K_{3}$ the set $\tau^{-1}(\{j\})$ contains one vertex with an odd index and one vertex with an even index (see Figure 4.2 (a)). So $\left\{v_{i}, v_{(i+2) \bmod 6}, v_{(i+4) \bmod 6}\right\} \cap \tau^{-1}(\sigma(x))=\left\{v_{i}\right\}$. Hence $w \sim v_{i}$ for every $w \in N(u) \cap A$. Then if $|N(u) \cap A| \geq 3$, this would create a copy of $\mathrm{H}_{2}$, depicted in Figure 4.8 (a). Thus $|N(u) \cap A| \leq 2$. 


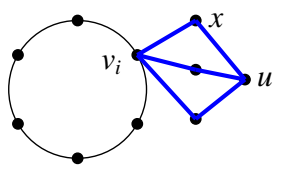

(a) $|N(u) \cap A| \geq 3 ; H_{2}$

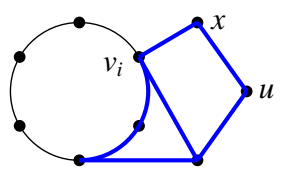

(b) $|N(u) \cap A|=2$, $\left|N(u) \cap A_{2,0}\right| \geq 1 ; H_{1}$

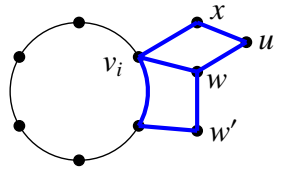

(c) $|N(u) \cap A|=2$, $\left|N(u) \cap A_{1,1}\right| \geq 1 ; H_{1}$

FigURE 4.8. $k=3$ and $|N(u) \cap A| \geq 2$.

Suppose that $|N(u) \cap A|=2$. If there was an adjacency from the set $\left\{v_{(i+2) \bmod 6}, v_{(i+4) \bmod 6}\right\}$ to $N(u) \cap A$, it would create a copy of $H_{1}$, as in Figure 4.8 (b). So $N(u) \cap A_{2,0}=\varnothing$. And adjacent pairs in $A_{1,1}$ form squares with their connecting pair in $H$. So if $w \in N(u) \cap A_{1,1}$, then there is $w^{\prime} \in N(w) \cap A_{1,1}$ such that, without loss of generality, $w^{\prime} \sim v_{i+1} \bmod 6$. But, as depicted in Figure 4.8 (c), this creates a copy of $H_{1}$. Thus it cannot be that that both $|N(u) \cap A|>1$ and $N(u) \cap\left(A_{2,0} \cup A_{1,1}\right) \neq \varnothing$. Since $x \in \bar{A} \subseteq A_{2,0} \cup A_{1,1}$ by assumption, we have that $N(u) \cap A=\{x\}$, and so $N(u) \cap(A \backslash \bar{A})=\varnothing$.

Now it suffices to check for all $w \in N(u) \backslash(H \cup\{x\})$ that $N(w) \cap \bar{A}=\varnothing$. Suppose that $u \in B_{1}$. Then $N(u) \cap H \subset\left\{v_{(i+1) \bmod 6}, v_{(i+3) \bmod 6}, v_{(i+5) \bmod 6}\right\}$ since $x \sim v_{i}$. Further suppose that $x \in A_{2,0}$, so without loss of generality $N(x) \cap H=\left\{v_{i}, v_{(i+2) \bmod 6}\right\}$. Then all three possible adjacencies for $u$ in $H$ are shown in Figure 4.9, with $u \sim v_{(i+1) \bmod 6}$ creating a copy of $H_{2}$, while $v \sim v_{(i+3)} \bmod 6$ or $v \sim v_{(i+5) \bmod 6}$ each creates a copy of $H_{1}$. Hence $x \notin A_{2,0}$.

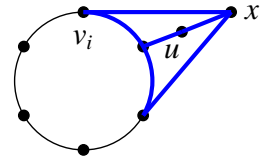

(a) $u \sim v_{(i+1) \bmod 6} ; H_{2}$

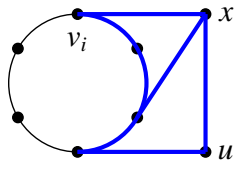

(b) $u \sim v_{(i+3) \bmod 6} ; H_{1}$

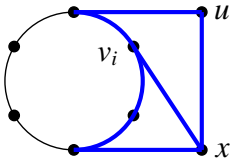

(c) $u \sim v_{(i+5) \bmod 6} ; H_{1}$

FIGURE 4.9. $k=3, u \in B_{1}$, and $x \in A_{2,0}$.

So $x \in \bar{A} \cap A_{1,1}$ which implies that $\sigma(x)=\{2\}$, and so $\sigma(u)=\{1\}$. Then without loss of generality let $i=2$. Note $x \sim v_{2}$ implies $u$ must be connected to a vertex in $H$ which has odd index. But $u \notin A$, so $u \nsim v_{1}$. Let $\{w\}=N(x) \cap A_{1,1}$, so it must be that $\sigma(w)=\{1\}$ and $w \sim v_{1}$. Then $u \sim v_{3}$ would create a copy of $H_{1}$, shown in Figure 4.10 (a), and so $u \sim v_{5}$. Now consider a vertex $z \in N(u) \backslash(H \cup\{x\})$, and suppose there exists $v_{z} \in N(z) \cap \bar{A}$. Then $d\left(u, v_{z}\right)=2$, so $\sigma\left(v_{z}\right)=\sigma(u)=\{1\}$. And by definition, we have that $\{v \in \bar{A}: \sigma(v)=\{1\}\} \subseteq A_{2,0}$, so $v_{z} \in A_{2,0}$ and the two vertices in $N\left(v_{z}\right) \cap H$ have images $\{1\}$ and $\{3\}$ under $\sigma$. Since $\sigma\left(v_{z}\right)=\sigma(u)$, vertices in $N\left(v_{z}\right) \cap H$ must have odd index. Thus $N\left(v_{z}\right) \cap H=\left\{v_{1}, v_{3}\right\}$. As shown in Figure 4.10 (b), $\left\{w, x, v_{z}, v_{1}, v_{2}, v_{3}\right\}$ is then a copy of $H_{1}$. So if $u \in B_{1}$ there cannot be any vertex $z \in N(u)$ with $N(z) \cap \bar{A} \neq \varnothing$. 


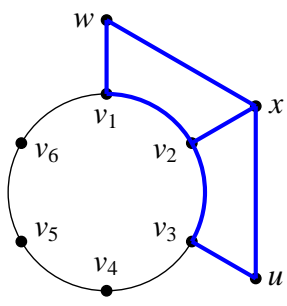

(a) $u \sim v_{3} ; H_{1}$

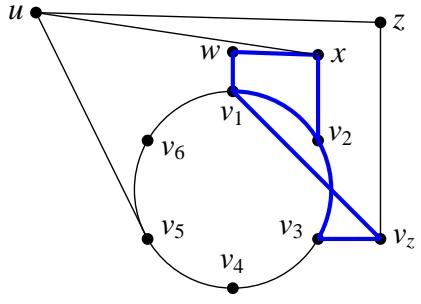

(b) $u \sim z \sim v_{z}, v_{z} \in \bar{A} ; H_{1}$

FIGURE 4.10. $k=3, u \in B_{1}$, and $x \in \bar{A} \cap A_{1,1}$.

Now assume that $u \in B_{2}$. Let $x=x_{u}$, and suppose that there is a vertex $w \in N(u)$ which has a vertex $x_{w} \in N(w) \cap \bar{A}$. Then there is a path of length 3 from $x_{u}$ to $x_{w}$, so $x_{u} \neq x_{w}$ and also $\sigma\left(x_{u}\right) \neq \sigma\left(x_{w}\right)$. Then they cannot both be in $A_{1,1}$, since $\sigma(v)=\{2\}$ for all $v \in \bar{A} \cap A_{1,1}$. Without loss of generality, let $x_{u} \in A_{2,0}$. Suppose that $x_{w} \in A_{1,1}$ and let $\{z\}=N\left(x_{w}\right) \cap A_{1,1}$. Then $\sigma\left(x_{w}\right)=\{2\}$ and without loss of generality we have that $x_{w} \sim v_{2}$ and $z \sim v_{1}$. Now $x_{u}$ must be adjacent to two vertices in $H$ with odd indices, one of which has image $\{3\}$ under $\tau$. And $\sigma\left(x_{u}\right)=\{1\}$, so $N\left(x_{u}\right) \cap H=\left\{v_{1}, v_{3}\right\}$. This is depicted in Figure 4.11 (a), where $\left\{v_{1}, v_{2}, v_{3}, x_{u}, x_{w}, z\right\}$ forms a copy of $H_{1}$. Suppose instead that $x_{w} \in A_{2,0}$. Then each $x_{w}$ and $x_{u}$ must be adjacent to a vertex whose image under $\tau$ is $\{3\}$. Without loss of generality assume that $x_{u} \sim v_{3}$ and $x_{w} \sim v_{6}$, and let $x_{u} \sim v_{j}$ and $x_{w} \sim v_{l}$ be the other two adjacencies. Then $j$ must be odd and $l$ must be even, and $\tau\left(v_{j}\right)=\sigma\left(x_{u}\right) \neq \sigma\left(x_{w}\right)=\tau\left(v_{l}\right)$. So either $j=1$ and $l=2$, or $j=5$ and $l=4$. By symmetry we may assume that $x_{u} \sim v_{1}$ and $x_{w} \sim v_{2}$, as in Figure 4.11 (b). But then $\left\{v_{1}, v_{2}, v_{3}, v_{6}, x_{u}, x_{w}\right\}$ forms a copy of $H_{1}$. Thus $N(w) \cap \bar{A}=\varnothing$ for all $w \in N(u)$.

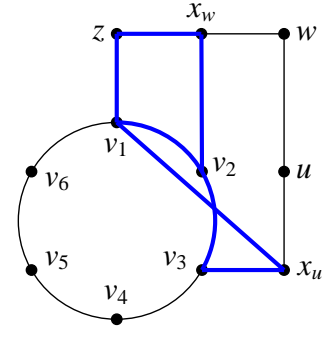

(a) $x_{w} \in A_{1,1} ; H_{1}$

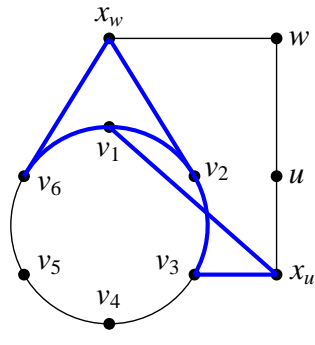

(b) $x_{w} \in A_{2,0} ; H_{1}$

Figure 4.11. $k=3, u \in B_{2}$, and $\left\{x_{u}, x_{w}\right\} \cap A_{2,0} \neq \varnothing$.

It follows immediately from Claims 4.11 and 4.12 that $\eta: G \rightarrow K_{3}$ is a homomorphism for all $k \geq 3$. And $\eta$ extends $\tau$, so $\eta_{\{l, j\}}$ extends $\tau_{\{l, j\}}$. Thus by Lemma 4.2, $\eta$ and $\eta_{\{l, j\}}$ are in distinct connected components of $\operatorname{Hom}\left(G, K_{3}\right)$, which is therefore disconnected. $\square$

The details of this proof suggests that it is only necessary to require that there be some cycle $C_{2 k} \subset G, k \geq 3$, such that $H^{\prime} \cap C_{2 k}=\varnothing$ for any subgraph $H^{\prime} \subset G$ with $H^{\prime} \in$ $\left\{H_{1}, H_{2}\right\}$. But even this leaves out many bipartite $G$ for which $\operatorname{Hom}\left(G, K_{3}\right)$ is disconnected. For example, consider the graph $G^{\prime}$ which is a circular ladder with six rungs. So $G^{\prime}$ is 
two disjoint copies of $C_{6}$ with respective vertices labeled consecutively as $v_{1}, \ldots, v_{6}$, and $w_{1}, \ldots w_{6}$, and additional edges $v_{i} \sim w_{i}$ for each $i$. Note that $G^{\prime}$ is bipartite, does not admit any folds, and every edge is contained in at least two copies of $H_{1}$. Define $\eta\left(v_{i}\right)=\{i \bmod 3\}$ and $\eta\left(w_{i}\right)=\{(i+1) \bmod 3\}$ for all $i$. Then let $\tau$ be the restriction of $\eta$ to the induced cycle on $\left\{v_{1}, \ldots, v_{6}\right\}$. So $r(\tau)=0$, and as before $\eta$ and $\eta_{\{l, j\}}$ are in distinct connected components of $\operatorname{Hom}\left(G^{\prime}, K_{3}\right)$ for any fixed pair $\{l, j\} \subset\{1,2,3\}$.

In the contrarian case of $Q_{3}$, again every edge is contained in multiple copies of $H_{1}$. The real issue, however, is that every 6-cycle in $Q_{3}$ is contained in either an induced copy of $H_{1}$ or an induced copy of $Q_{3} \backslash\{v\}$ for some $v \in Q_{3}$. Both of these graphs fold to an edge, so $\operatorname{Hom}\left(H_{1}, K_{3}\right)$ and $\operatorname{Hom}\left(Q_{3} \backslash\{v\}, K_{3}\right)$ are each connected. But then any distinct 0-cells $\tau_{1}, \tau_{2} \in \operatorname{Hom}\left(C_{6}, K_{3}\right)$ which extend to all of $Q_{3}$ must first extend to either $H_{1}$ or $Q_{3} \backslash\{v\}$, where their extensions are necessarily in a single connected component.

\section{5. Ном $\left(G(n, p), K_{m}\right)$}

The topological connectivity of random hom-complexes has been studied previously under the guise of the Neighborhood Complex of $G(n, p)$, the Erdős-Rényi model for a random graph. $G(n, p)$ is the probability space of graphs on $n$ vertices where each edge is inserted independently with probability $p=p(n)$. Kahle [9] showed that the connectivity of $\mathcal{N}(G(n, p))$ is concentrated between $1 / 2$ and $2 / 3$ of the expected value of the largest clique in $G(n, p)$, and also obtained asymptotic bounds on the number of dimensions with non-trivial homology. As we discussed earlier, $\mathcal{N}(G(n, p))$ is homotopy equivalent to $\operatorname{Hom}\left(K_{2}, G(n, p)\right)$. Here we take the opposite perspective and consider the random polyhedral complex $\operatorname{Hom}\left(G(n, p), K_{m}\right)$.

The major benefit of utilizing $D(G)$ in a generalization of the Cukić-Kozlov Theorem is that $k$-cores have been well-studied in random graphs models. We say that $G(n, p)$ has property $\mathcal{P}$ with high probability if $\lim _{n \rightarrow \infty} \operatorname{Pr}[G(n, p) \in \mathcal{P}]=1$. A property $\mathcal{P}$ is said to have a sharp threshold $\hat{p}=\hat{p}(n)$ if for all $\epsilon \rightarrow \infty$

$$
\lim _{n \rightarrow \infty} \operatorname{Pr}[G(n, p) \in \mathcal{P}]=\left\{\begin{array}{ll}
0 & \text { if } p \leq(1-\epsilon) \hat{p} \\
1 & \text { if } p \geq(1+\epsilon) \hat{p}
\end{array}\right\}
$$

When $p=c / n$ for constant $c>0$, Pittel, Spencer and Wormwald [13] showed that the existence of a $k$-core in $G(n, p)$ has a sharp threshold $c=c_{k}$ for $k \geq 3$, and that asymptotically $c_{k}=k+\sqrt{k \log k}+O(\log k)$. Approximate values for small $k$ are known, such as $c_{3} \approx 3.35, c_{4} \approx 5.14, c_{5} \approx 6.81$. When $k=2$, the existence of cycles has a one-sided sharp threshold at $c=1$. For $c>1$, indeed $\operatorname{Pr}[D(G(n, c / n)) \geq 2] \rightarrow 1$. However, for all $0<c<1$ there is a constant $0<f(c)<1$ for which $\operatorname{Pr}[D(G(n, c / n)) \geq 2] \rightarrow f(c)$. To simplify this issue, define $c_{k}$ for all $k \geq 2$ by

$$
c_{k}:=\sup \left\{c>0: \lim _{n \rightarrow \infty} \operatorname{Pr}[D(G(n, c / n) \geq k]=0\}\right.
$$

In particular $c_{2}=0$, and for $k \geq 3$ these are precisely the sharp thresholds mentioned above.

By applying Theorem 2.8 to these thresholds, we then immediately obtain lower bounds on the topological connectivity of $\operatorname{Hom}\left(G(n, p), K_{m}\right)$. For notational convenience, define $M(n, c, m):=\operatorname{conn}\left[\operatorname{Hom}\left(G(n, c / n), K_{m}\right)\right]$.

Theorem 5.1. If $k \geq 2$ and $p=c / n$ with $c<c_{k+1}$, then for all $m \geq 3$

$$
\lim _{n \rightarrow \infty} \operatorname{Pr}[M(n, c, m) \geq m-k-2]=1
$$


Theorem 2.8 does not require that the input graph be connected, and in fact $G(n, p)$ will be disconnected when $p=c / n$. Let the disjoint connected components of $G(n, p)$ be $G_{0}, G_{1}, \ldots, G_{t}$, ordered from most vertices to least. For $c>1$, with high probability $G_{0}$ is a giant component containing more than half the vertices, and $G_{i}$ is either an isolated vertex, a tree, or a unicyclic graph for $i \geq 1$. So

$$
\operatorname{Hom}\left(G(n, c / n), K_{m}\right)=\operatorname{Hom}\left(\coprod_{0}^{t} G_{i}, K_{m}\right)=\prod_{i=0}^{t} \operatorname{Hom}\left(G_{i}, K_{m}\right)
$$

And thus

$$
M(n, c, m)=\min _{0 \leq i \leq t}\left\{\operatorname{conn}\left[\operatorname{Hom}\left(G_{i}, K_{m}\right)\right]\right\}
$$

For $G_{i}=\left\{v_{i}\right\}, \operatorname{Hom}\left(G_{i}, K_{m}\right)=\Delta^{2}$, which is contractible. If a finite connected graph $G_{i}$ is a tree, then it folds to a single edge and $\operatorname{Hom}\left(G_{i}, K_{m}\right) \simeq \operatorname{Hom}\left(K_{2}, K_{m}\right) \simeq S^{m-2}$. Finally, if $G_{i}$ is a finite unicyclic graph, then it folds to its cycle $C_{n}$. If $n=4$, then $C_{4}$ folds further to $K_{2}$ and $\operatorname{Hom}\left(G_{i}, K_{m}\right) \simeq S^{m-2}$. Thus, with high probability we have

$$
\operatorname{Hom}\left(G(n, c / n), K_{m}\right)=\left(\Delta^{2}\right)^{t_{1}} \times\left(S^{m-2}\right)^{t_{2}} \times \operatorname{Hom}\left(G_{0}, K_{m}\right) \times \prod_{j=1}^{t_{3}} \operatorname{Hom}\left(C_{n_{j}}, K_{m}\right)
$$

where $t_{1}=$ the number of isolated vertices, $t_{2}=$ the number of trees and unicyclic components whose cycle is $C_{4}, t_{3}=t-\left(t_{1}+t_{2}\right)$, and $n_{j} \neq 4$ for all $j$.

When $n_{j}=3, \operatorname{Hom}\left(C_{n_{j}}, K_{m}\right)=\operatorname{Hom}\left(K_{3}, K_{m}\right)$, which is a wedge of $(m-3)$-spheres [2]. Kozlov also computed the homology of $\operatorname{Hom}\left(C_{n}, K_{m}\right)$ for all $n \geq 5, m \geq 4$ in [10]. In particular, for all $m \geq 4$ :

$$
\begin{aligned}
& \text { conn }\left[\operatorname{Hom}\left(C_{2 r+1}, K_{m}\right)\right]=m-4 \text { for } r \geq 1 \\
& \text { conn }\left[\operatorname{Hom}\left(C_{2 r}, K_{m}\right)\right]=m-3 \text { for } r \geq 2
\end{aligned}
$$

Note that the cases $n=3$ and $n=4$, which yield spheres, are consistent with these values. And the threshold for all small components to be isolated vertices is $p=\frac{\log n}{4 n}$, so for $c<c_{k+1}, k \geq 2$, with high probability there is some $i \geq 1$ such that

$$
\operatorname{conn}\left[\operatorname{Hom}\left(G_{i}, K_{m}\right)\right] \in\{m-3, m-4\}
$$

This provides an upper bound for $M(n, c, m)$, and so when $m \geq 4$ we improve Theorem 5.1 to the following:

Theorem 5.2. If $k \geq 2$ and $p=c / n$ with $c<c_{k+1}$, then for all $m \geq 4$

$$
\lim _{n \rightarrow \infty} \operatorname{Pr}[m-k-2 \leq M(n, c, m) \leq m-3]=1
$$

For fixed $m$, the lower bound decreases as $c$ gets bigger, and the gap between upper and lower bounds becomes worse. We expect that conn $\left[\operatorname{Hom}\left(G_{0}, K_{m}\right)\right]$ should decrease as $D\left(G_{0}\right)$ increases and the giant component becomes more highly connected. However, the lower bound in Theorem 2.8 is not tight in general. For example, the complete bipartite graph $K_{i, j}$ folds to an edge, $\operatorname{so} \operatorname{Hom}\left(K_{i, j}, K_{m}\right) \simeq S^{m-2}$. But $D\left(K_{i, j}\right)=\min \{i, j\}$, so Theorem 2.8 yields conn $\left[\operatorname{Hom}\left(K_{i, j}, K_{m}\right)\right] \geq m-\min \{i, j\}-2$. If $i, j \geq 2$, then this bound is not tight, and for large $i, j$ it can be arbitrarily bad.

To sharpen these results, we turn to examining the chromatic number of $G(n, c / n)$. Similar to the thresholds for the appearance of $k$-cores, Achlioptas and Friedgut [1] showed that there is a sharp threshold sequence $d_{k}(n)$ such that for any $\epsilon>0$,

$$
c<(1-\epsilon) d_{k}(n) \Longrightarrow \lim _{n \rightarrow \infty} \operatorname{Pr}[\chi(G(n, c / n)) \leq k]=1
$$




$$
c>(1+\epsilon) d_{k}(n) \Longrightarrow \lim _{n \rightarrow \infty} \operatorname{Pr}[\chi(G(n, c / n)) \geq k+1]=1
$$

The convergence of the $d_{k}(n)$ remains an open problem, but Coja-Oghlan and Vilenchik [4] improved the lower bound on $\liminf d_{k}(n)$ to within a small constant of the upper bound for $\limsup _{n \rightarrow \infty} d_{k}(n)$. In particular, for $o_{k}(1)$ a term which goes to 0 as $k$ becomes large,

$$
\begin{gathered}
d_{k}^{-}:=\liminf _{n \rightarrow \infty} d_{k}(n) \geq 2 k \log k-\log k-2 \log 2-o_{k}(1) \\
d_{k}^{+}:=\limsup _{n \rightarrow \infty} d_{k}(n) \leq 2 k \log k-\log k-1+o_{k}(1)
\end{gathered}
$$

Then as $k$ increases, the difference $\left|d_{k}^{+}-d_{k}^{-}\right|$approaches $2 \log 2-1 \approx 0.39$. And in the context of hom-complexes it is immediate that with high probability

$$
\operatorname{Hom}\left(G(n, c / n), K_{m}\right)=\varnothing \text { for } c>d_{m}^{+} \text {and } \operatorname{Hom}\left(G(n, c / n), K_{m}\right) \neq \varnothing \text { for } c<d_{m}^{-}
$$

For $m=3$, we can then evaluate the connectivity of $\operatorname{Hom}\left(G(n, c / n), K_{3}\right)$ for all $c>0$, excluding the gap between $d_{3}^{-}$and $d_{3}^{+}$.

Theorem 5.3. For $1 \leq c<d_{3}^{-}$, $\operatorname{Hom}\left(G(n, c / n), K_{3}\right)$ is disconnected with high probability.

Proof of Theorem 5.3. By the definition of $d_{3}^{-}$and the existence of odd cycles with high probability for $c \geq 1, \chi(G(n, c / n))=3$. Then by Theorem $4.6 \operatorname{Hom}\left(G(n, c / n), K_{3}\right)$ is disconnected with high probability. $\square$

When $0<c<1$, all connected components are isolated vertices, trees, or unicyclic graphs with high probability. If there is a component which contains edges and does not fold to a single edge, then $\operatorname{Hom}(G(n, c / n)$ will be disconnected. But if every connected component of $G(n, c / n)$ is an isolated vertex or folds to an edge, then $\operatorname{Hom}\left(G(n, c / n), K_{3}\right)$ is connected. In the latter case, specifically

$$
\operatorname{Hom}\left(G(n, c / n), K_{3}\right)=\left(\Delta^{2}\right)^{t_{1}} \times \mathbb{T}^{t_{2}}
$$

where $t_{1}=$ number of isolated vertices, and $t_{2}=$ the number of connected components which contain at least one edge. And $t_{2}>0$ with high probability for $p=c / n$, so the complex will not be contractible, and $M(n, c, 3)=0$.

Theorem 5.4. For $0<c<1, c^{\prime}=\frac{1}{2} \log (1-c)+\frac{c}{2}+\frac{c^{2}}{4}+\frac{c^{4}}{8}$,

$$
\lim _{n \rightarrow \infty} \operatorname{Pr}[M(n, c, 3)=-1]=1-e^{c^{\prime}} \text { and } \lim _{n \rightarrow \infty} \operatorname{Pr}[M(n, c, 3)=0]=e^{c^{\prime}}
$$

Proof of Theorem 5.4. If $G$ is a connected unicyclic graph whose cycle is $C_{l}$ for $l \neq 4$, then $G$ folds to $C_{l}$ and $\operatorname{Hom}\left(G, K_{3}\right) \simeq \operatorname{Hom}\left(C_{l}, K_{3}\right)$, which is disconnected. So if $G(n, c / n)$ does not contain any cycle $C_{l}$ for $l \neq 4$, then all components of $G(n, c / n)$ will be isolated vertices or will fold to an edge. For a fixed $l$, the number of $l$-cycles in $G(n, c / n)$ approaches a limiting Poisson distribution with mean $\frac{c^{l}}{2 l}$, and so

$$
\operatorname{Pr}\left[C_{l} \not \subset G(n, c / n) \text { for all } l \neq 4\right] \rightarrow \exp \left\{-\frac{c^{3}}{6}-\sum_{l=5}^{\infty} \frac{c^{l}}{2 l}\right\}
$$

For $c<1$, the sum in the exponent converges to $c^{\prime}=\frac{1}{2} \log (1-c)+\frac{c}{2}+\frac{c^{2}}{4}+\frac{c^{4}}{8}$. Thus $\operatorname{Pr}[M(c, n, 3)=0] \rightarrow e^{c^{\prime}}$ and $\operatorname{Pr}[M(c, n, 3)=-1] \rightarrow 1-e^{c^{\prime}}$. 


\section{Further Questions}

The biggest question left unanswered is that of bounding conn $\left[\operatorname{Hom}\left(G_{0}, K_{m}\right)\right]$ from above. We expect that $\operatorname{conn}\left[\operatorname{Hom}\left(G_{0}, K_{m}\right)\right]$ remains close to $m-k-2$ when this bound makes sense, that $M(n, c, m)=\operatorname{conn}\left[\operatorname{Hom}\left(G_{0}, K_{m}\right)\right]$, and that $M(n, c, m)$ is a non-increasing function for fixed $m$, with high probability. However, exhibiting non-trivial homology classes in $\operatorname{Hom}(G, H)$ is difficult in general. In Section 4 we showed that disconnected components can be lifted via subgraphs, which can be viewed as lifting non-trivial 0cycles, but there is no known analogous result for lifting higher dimensional non-trivial cycles.

We also expect that $M(n, c, m)$ increases monotonically when $c$ is fixed and $m$ is increasing, but this is not true in general for a fixed input graph. For instance, consider again our favorite counter example, $Q_{3}$. We noted in Section 4 that conn $\left[\operatorname{Hom}\left(Q_{3}, K_{3}\right)\right] \geq 0$. And by Theorem 2.8, conn $\left[\operatorname{Hom}\left(Q_{3}, K_{5}\right)\right] \geq 0$. But for $m=4$, there are colorings of $Q_{3}$ in which each color class is a pair of antipodal corners. In such a coloring, every vertex is adjacent to one vertex from each of the other three color class, so it represents an isolated 0-cell in $\operatorname{Hom}\left(Q_{3}, K_{4}\right)$. Thus conn $\left[\operatorname{Hom}\left(Q_{3}, K_{4}\right)\right]=-1$. In this example with $Q_{3}$, the problem seems to occur when $m \leq D(G)$, and it is plausible that conn $\left[\operatorname{Hom}\left(G, K_{m}\right)\right]$ is either increasing or non-decreasing for $m \geq D(G)+1$, if $G$ is a fixed graph which cannot be reduced via folds. This, however, remains an open question.

In the random setting, considering $m \leq D(G)$ presents a serious roadblock to evaluating $M(n, c, m)$ for all pairs of $c$ and $m$. Since the $d_{k}^{ \pm}$grow much faster than the $c_{k}$, there are intervals where $D(G(n, c / n))$ is much larger than $\chi(G(n, c / n))$. For example, Molloy [12] pointed out that $c_{40} \approx 52.23$ while $d_{19}>53.88$. So if $c=53$, with high probability $D(G(n, c / n))=40$, but $m$ can be as small as 19 before $\operatorname{Hom}\left(G(n, c / n), K_{m}\right)$ becomes empty. When $m \leq D(G)$ the lower bound from Theorem 2.8 provides no information, and new methods are required.

A different direction altogether would be to indulge in a closer examination of precise numerical estimates on the Betti numbers and Euler characteristic of $\operatorname{Hom}\left(G(n, c / n), K_{m}\right)$. Cukić and Kozlov's [7] work on cycles in hom-complexes makes the case that $m=3$ a tantalizingly tractable place to start this type of investigation.

\section{REFERENCES}

[1] Dimitris Achlioptas and Ehud Friedgut. A sharp threshold for $k$-colorability. Random Structures Algorithms, 14(1):63-70, 1999.

[2] Eric Babson and Dmitry N. Kozlov. Complexes of graph homomorphisms. Israel J. Math., 152:285-312, 2006.

[3] Luis Cereceda, Jan van den Heuvel, and Matthew Johnson. Mixing 3-colourings in bipartite graphs. European J. Combin., 30(7):1593-1606, 2009.

[4] Amin Coja-Oghlan and Dan Vilenchik. Chasing the $k$-colorability threshold. In 2013 IEEE 54th Annual Symposium on Foundations of Computer Science-FOCS 2013, pages 380-389. IEEE Computer Soc., Los Alamitos, CA, 2013.

[5] Peter Csorba. Non-Tidy Spaces and Graph Colorings. PhD thesis, ETH Zürich, 2005.

[6] Sonja Lj. Čukić and Dmitry N. Kozlov. Higher connectivity of graph coloring complexes. Int. Math. Res. Not., (25):1543-1562, 2005.

[7] Sonja Lj. Čukić and Dmitry N. Kozlov. The homotopy type of complexes of graph homomorphisms between cycles. Discrete Comput. Geom., 36(2):313-329, 2006.

[8] Alexander Engström. A short proof of a conjecture on the connectivity of graph coloring complexes. Proc. Amer. Math. Soc., 134(12):3703-3705 (electronic), 2006.

[9] Matthew Kahle. The neighborhood complex of a random graph. J. Combin. Theory Ser. A, 114(2):380-387, 2007. 
[10] Dmitry N. Kozlov. Cohomology of colorings of cycles. Amer. J. Math., 130(3):829-857, 2008.

[11] L. Lovász. Kneser's conjecture, chromatic number, and homotopy. J. Combin. Theory Ser. A, 25(3):319-324, 1978.

[12] Michael Molloy. A gap between the appearances of a $k$-core and a $(k+1)$-chromatic graph. Random Structures Algorithms, 8(2):159-160, 1996.

[13] Boris Pittel, Joel Spencer, and Nicholas Wormald. Sudden emergence of a giant $k$-core in a random graph. J. Combin. Theory Ser. B, 67(1):111-151, 1996.

[14] Carsten Schultz. Small models of graph colouring manifolds and the Stiefel manifolds $\operatorname{Hom}\left(C_{5}, K_{n}\right) . J$. Combin. Theory Ser. A, 115(1):84-104, 2008.

Department of Mathematics, The Ohio State University 